\title{
Kinderradiologisches Wissen für Allgemeinradiologen und -radiologinnen - ein Veranstaltungsrückblick
}

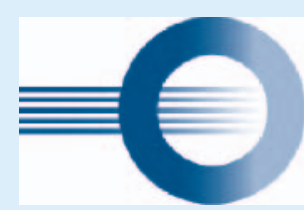

AC Pädiatrische Radiologie

in der Deutschen Röntgengesellschaft

Liebe Kolleginnen und Kollegen,

am 19. und 20. März 2021 fand die kinderradiologische Fortbildung der Arbeitsgemeinschaft Pädiatrische Radiologie der Deutschen Röntgengesellschaft als digitale Veranstaltung statt. Als Leiter der beiden Kinder- und Jugendradiologien in Köln hatten wir die Ehre, das wissenschaftliche Programm auszurichten.

Die Veranstaltung war der Auftakt einer modularen Veranstaltungsreihe über mehrere Jahre, die von der AG Pädiatrische Radiologie konzipiert wurde, um insbeson- dere Radiologinnen und Radiologen mit wenig oder fehlender Erfahrung in der Bildgebung bei Kindern und Jugendlichen eine Möglichkeit zur Weiterbildung auf dem Gebiet der Kinder- und Jugendradiologie anbieten zu können.

Der modulare Ansatz basiert auf der Organradiologie und umfasst sämtliche bildgebende Modalitäten.

Bei der Premiere der Modulveranstaltung wurde das Thema „Thorakale Bildgebung bei Kindern und Jugendlichen“ gewählt. Ausgenommen war aufgrund ihrer Komple-

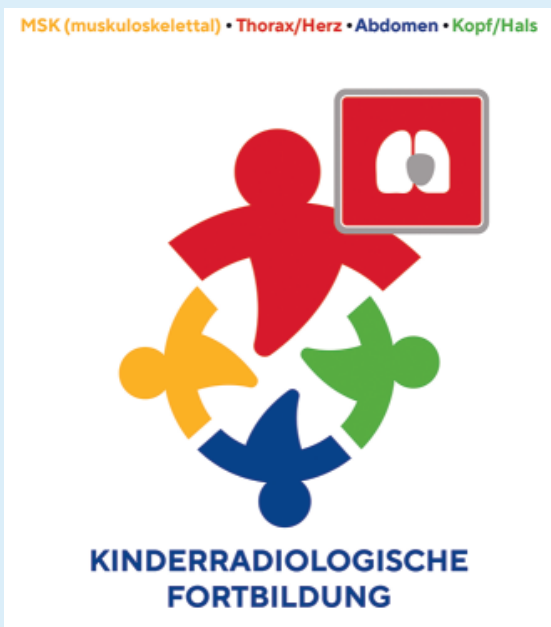

xität die kardiale Bildgebung, welche intensiv in einer Folgeveranstaltung berücksichtigt wird. 
Am Freitagnachmittag bildeten die Grundlagen der thorakalen Bildgebung beim Säugling und Kleinkind den Auftakt.

Mit dem ersten Block wurden die Untersuchungstechniken sowie Katheter, Tuben und Drainageversorgung des Früh- und Reifgeborenen vorangestellt. Dabei wurden insbesondere Komplikationen, Fehlplatzierungen und die kindliche Anatomie erörtert. Die Möglichkeiten schneller und präziser MRT-Sequenzen unter Berücksichtigung der kindlichen Physiologie fanden in einem weiteren Vortrag Platz.

Die zweite Sitzung beschäftigte sich mit den angeborenen Fehlbildungen. Interdisziplinär wurde sich zwischen dem Fach Kinderchirurgie und Kinderradiologie ausgetauscht. Die Notwendigkeit einer präzisen und möglichst wenig belastenden Bildgebung zur korrekten Klassifikation von anatomischen Anlagestörungen wurde im Themenblock offen und hochwertig diskutiert.

Der letzte Block des Freitags war den Besonderheiten der Früh- und Extremfrühgeborenen gewidmet. Bei der Rednerauswahl wurde Wert auf die Möglichkeit eines interdisziplinären Diskurses gelegt. Insbesondere die medizinische Versorgung der Frühgeborenen stellt eine große Herausforderung an alle behandelnden Ärztinnen und Ärzte dar, sodass auch die Radiologie hier ganz besonders gefordert ist. Strahlenschutz und schonender Umgang mit den Frühchen - das war der Tenor, der sich durch alle Vorträge zog.

Der Samstag startete mit dem Block „Onkologie“. Die verschiedenen Facetten primärer und sekundärer Tumoren des Thorax wurden aufgezeigt. Möglichkeiten der MRT zur Beurteilung von Lungenmetastasen im Vergleich zur Computertomografie wurden intensiv diskutiert. Die Bildgebung der pulmonalen Komplikationen intensivierter onkologischer Therapie, wie zum Beispiel Infektionen des immunsupprimierten Pa- tienten beziehungsweise der immunsupprimierten Patientin, wurde vermittelt.

Entzündliche Veränderungen der Lungen bildeten die Grundlage der nächsten Sitzung. Dass nicht jede Lungenentzündung einer Röntgenaufnahme bedarf und alternative Methoden wie die Sonografie eingesetzt werden können, wurde mit dem nächsten Vortrag dem Auditorium vermittelt. Neben den bakteriellen Infektionen wurde auch die Thematik „COVID-19“ beleuchtet. Neben der Primärinfektion stellt eine Sekundärkomplikation, das PIMS, bei Kindern eine diagnostische und therapeutische Herausforderung dar.

Der letzte Block des Samstags widmete sich dem kindlichen Thoraxtrauma. Das Spektrum der Vorträge reichte von nichtakzidentellen Verletzungen bis zum Thoraxtrauma im Rahmen des Polytraumas. Die Sitzung wurde mit einem Vortrag zu Möglichkeiten und Limitationen der Sonografie pünktlich beendet.

An der Veranstaltung nahmen 171 Personen teil, davon auch einige medizinischtechnische Radiologieassistenten und -assistentinnen. Aus einer Live-Umfrage wurde bekannt, dass $57 \%$ der Teilnehmerinnen und Teilnehmer überwiegend keine Kinder und Jugendlichen versorgen, sodass das Ziel der Veranstaltung, kinderradiologisches Wissen Allgemeinradiologen zu vermitteln, erreicht werden konnte.

Auch eine Beteiligung der Industriepartner konnte verwirklicht werden - die Diskussion von Dosisreferenzwerten, deren Dokumentation, Überprüfung und Einhaltung zeigte die verschiedenen Möglichkeiten der Anwendung des ALARA-Prinzips, insbesondere beim jungen und sehr jungen $\mathrm{Pa}$ tienten, auf. Aufgeworfene Fragen im Chat und Kommentare des Auditoriums wurden in den teils lebhaften Diskussionen interaktiv und offen besprochen. Die Inhalte zeigten auf, dass bei der Bildgebung viele Teil- aspekte berücksichtigt werden müssen, die bei erwachsenen Patienten und Patientinnen nur eine geringe oder keine Rolle spielen. Insbesondere die Bildgebung des polytraumatisierten Kindes sorgte für eine kontroverse Diskussion darüber, wie ausgedehnt die Bildgebung durchzuführen ist. Die Anwendung kinderspezifischer Untersuchungsprotokolle und Nutzung strahlensparender Algorithmen wurde einhellig empfohlen. Zusammenfassend konnte von den Rednern und Rednerinnen festgestellt werden, dass das polytraumatisierte Kind Anspruch hat, von Kindermedizinern/ -medizinerinnen (Kinderchirurgen/-chirurginnen, -traumatologen/-traumatologinnen, -radiologen/-radiologinnen und Pädiatern/Pädiaterinnen) mitbetreut zu werden.

Im März 2022 soll die curriculare Veranstaltung in der Landeshauptstadt von Niedersachsen ihre Fortsetzung finden - verantwortlich werden Frau Prof. Dr. Diane Miriam Renz, Leiterin der Pädiatrischen Radiologie des Instituts für Diagnostische und Interventionelle Radiologie der Medizinischen Hochschule Hannover, sowie Herr Dr. Jürgen Weidemann, Chefarzt der Klinik für Kinderradiologie, Ultraschall und Computertomografie des Kinder- und Jugendkrankenhauses Auf der Bult, sein. Inhalt dieser Veranstaltung wird die abdominale Radiologie des Kindes und Jugendlichen sein.

Die wissenschaftliche Leitung der diesjährigen Veranstaltung übergibt hiermit den Staffelstab und wünscht Prof. Renz und Dr. Weidmann bereits jetzt bei der Planung und Durchführung viel Erfolg!

Ihre

Privatdozentin Dr. med. Friederike Körber

Dr. med. M. Stenzel

Professor Dr. med. Jürgen Schäfer

(Vorsitzender der Arbeitsgemeinschaft Pädiatrische Radiologie) 